\title{
Vestibular schwannoma: 825 cases from a 25-year experience
}

\author{
Ricardo Ferreira Bento', Mariana Hausen Pinna², Rubens Vuono de Brito Neto³.
}

\author{
1) Full Professor. Head of Clinical Otolaryngology, University of São Paulo School of Medicine. Chief of Otology, HCFMUSP. \\ 2) Specialist ENT. Physician Group Otology HCFMUSP \\ 3) Full Professor, Division of Clinical Otolaryngology, HCFMUSP. \\ Institution: Hospital das Clínicas da Faculdade de Medicina da Universidade de São Paulo. \\ São Paulo / SP - Brazil. \\ Mailing address: Ricardo Ferreira Bento - Av. Dr. Enéas de Carvalho Aguiar 255 - Sala 6167 - São Paulo / SP - Brazil - Zip code: $05403-000$ - Telephone: (+55 11) \\ 2661-6288 - E-mail: rbento@ gmail.com \\ Article received in April 16, 2012. Article approved in July 31, 2012.
}

\section{SUMMARY}

Introduction: Acoustic nerve tumors have been recognized as a clinico-pathologic entity for at least 200 years, and they represent $90 \%$ of cerebellopontine angle diseases. Histologically, the tumors are derived from Schwann cells of the myelin sheath, with smaller tumors consisting of elongated palisade cells, while in large tumors, cystic degeneration can be found in the central areas, possibly due to deficient vascularization. We retrospectively reviewed 825 cases of vestibular schwannomas, reported between January 1984 and August 2006, in which the patients underwent surgery to remove the tumor.

Objective: To evaluate signs, symptoms, aspects of clinical diagnosis, including the results of audiological and imaging studies, and surgical techniques and complications.

Methods: A retrospective chart review. The medical records of all patients undergoing surgical treatment for schwannoma during the period indicated were reviewed.

Results and Conclusion: Hearing loss was the first symptom reported in almost all cases, and tumor size was not proportional to the impairment of the auditory threshold. The surgical techniques allowed safe preservation of facial function. In particular, the retrolabyrinthine route proved useful in small tumors, with 50\% preservation of hearing

Keywords: neuroma; acoustic; vestibulocochlear nerve diseases; cranial nerve neoplasms.

\section{INTRODUCTION}

Vestibulocochlear nerve neoplasms have been recognized as a distinct clinical and pathological entity for at least 200 years, and account for approximately $90 \%$ of all conditions affecting the cerebellopontine angle. The most appropriate term for these tumors is vestibular schwannoma $(1,2)$, although the vast majority of physicians refer to them as acoustic neuroma or neurinoma.

The first observation of a tumor of the acoustic nerve was made during an autopsy in 1777 by Eduard Sandifort, Professor of Anatomy at Leiden University (3). As the name implies, schwannomas are histologically derived from Schwann cells. Small schwannomas consist of elongated, palisade cells, whereas larger tumors also exhibit central cystic degeneration, possibly due to deficient vascularization. Unlike amputation neuromas, which contain a tangle of nerve fibers, schwannomas contain no such fibers (4).

The etiology of vestibularschwannomas is unknown. Cushing (1917) and Revilla (1948) believed trauma was a plausible explanation, as some observations apparently associated occipital trauma with tumors of the cerebellopontine angle $(5,6)$.
The finding that symptoms are sometimes exacerbated during pregnancy has elicited the hypothesis that hormonal mechanisms may be involved in the genesis or progression of these tumors $(3,7)$. A hereditary component is reasonably unlikely, except in the unquestionably inherited cases of schwannoma that present as part of the complex of neurofibromatosis type 2 .

Knowledge of the embryonic development of CN VIII constitutes the foundation of pathogenetic studies that have, on the one hand, provided an understanding of the underlying mechanisms of formation of these nerve tumors and, on the other, shown why of all the cranial nerves, the eighth pair is affected. The most widely accepted hypothesis is the embryonic theory of opposing distal and peripheral fibers that meet at the level of the internal auditory canal to give rise to the vestibulocochlear nerve.

In the vast majority of cases, the upper (vestibular) branch of the vestibulocochlear nerve is predisposed to the development of vestibular schwannomas and, in most cases, the tumor arises from the back of the internal auditory canal. Rarely, schwannomas may originate from the cochlear branch of CN VIII $(7,8,9)$. 


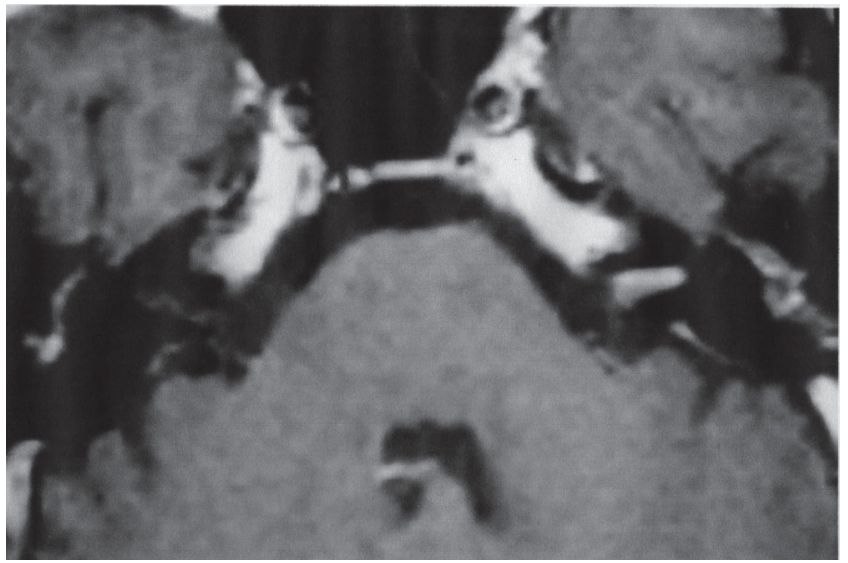

Figure 1. Tumor grade I.

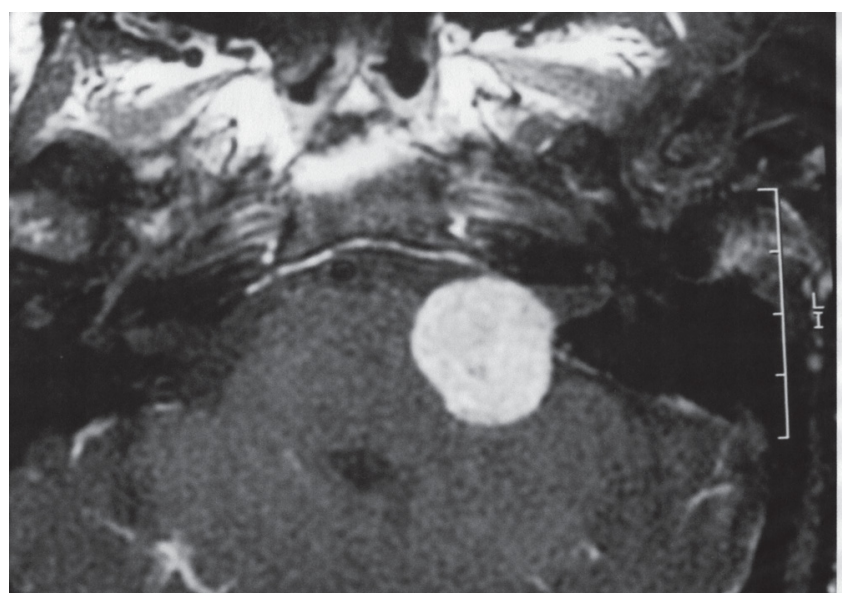

Figure 3. Tumor grade III.

Several studies have attempted to find an association between mobile phone use and the incidence of vestibular schwannoma. A multicenter European case-control study found no increase in the incidence rate of this tumor, nor did an investigation carried out between 2000 and 2004 in Japan. Therefore, there is currently no evidence confirming that cellular phone use could increase the incidence of vestibular schwannoma $(10,11)$.

Progress in the diagnosis of vestibular schwannoma has played a significant role in improving treatment outcomes. Early diagnoses are currently due to a spirit of enquiry that is almost exclusive to the field of otology. des:

Vestibular schwannomas are classified into 4 gra-

Grade I: exclusively intracanalicular tumor (Figure 1).

Grade II: tumor extending into the posterior fossa, with or without an intracanalicular component, without touching the brainstem (Figure 2).

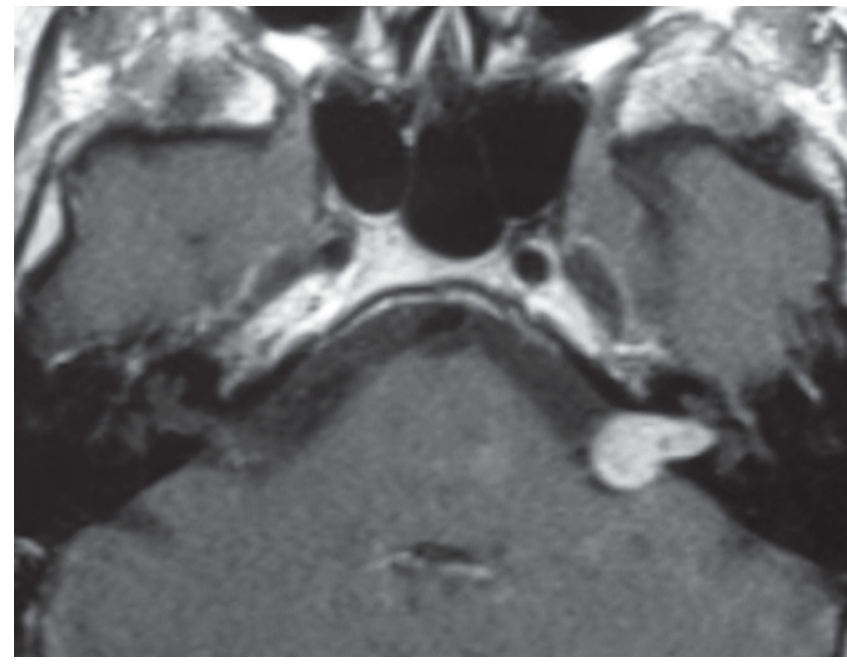

Figure 2. Tumor grade II.

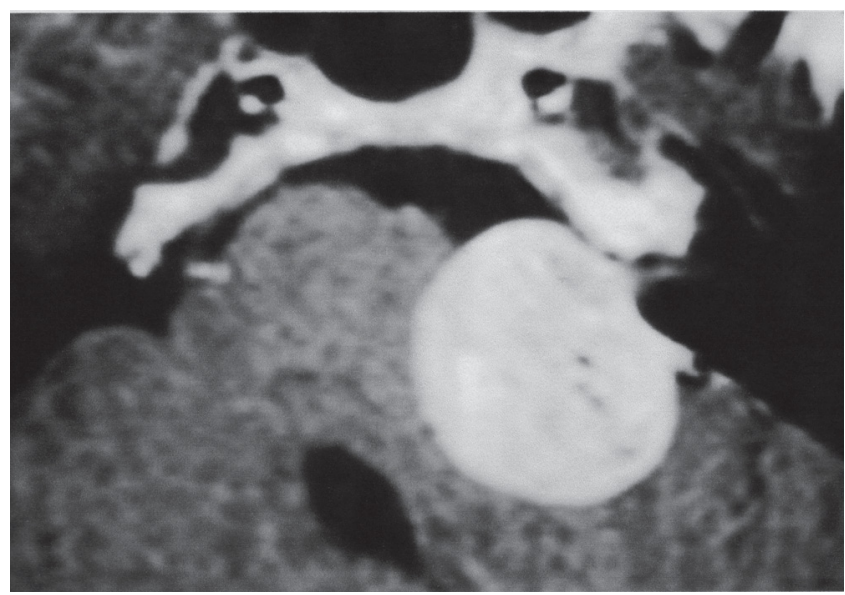

Figure 4. Tumor grade IV.

Grade III: tumor extending into the posterior fossa, compressing the brainstem, but not shifting it from the midline (Figure 3).

Grade IV: tumor extending into the posterior fossa, compressing the brainstem, and shifting it from the midline (Figure 4).

In his 1917 monograph, Cushing (5) provided an outstanding description of the usual sequence of onset and progression of the signs and symptoms of vestibular schwannoma:

- Auditory and labyrinthine symptoms.

- Occipitofrontal pain, followed by suboccipital discomfort.

- Incoordination and instability of cerebellar origin.

- Signs of adjacent cranial nerve involvement.

- Raised intracranial pressure, with papilledema and its consequences.

- Dysarthria, dysphagia, and, finally, cerebellar seizures and respiratory distress. 
In view of the currently available diagnostic and therapeutic armamentarium, this sequence now rarely ever reaches its end. However, it provides an important benchmark for disease staging.

In Brazil, the clinical signs, diagnosis, and surgical treatment of vestibular schwannoma have been studied by a number of groups (12-18). Their main findings are described in this introductory section.

The main symptom is hearing loss, often associated with tinnitus, due to compression of the cochlear nerve and disturbances in cochlear vascularization. This vascular mechanism explains the possibility of sudden, atypical, fluctuant hearing loss (19), often presenting with audiometry features suggesting peripheral involvement.

Other signs and symptoms may also be present, such as vertigo, dizziness, headache, hypoesthesia, and palsies. Clinical presentation is not always proportional to tumor size.

Classically, vestibularschwannomas are most often diagnosed around the fifth decade of life. Most authors report a clear female preponderance.

Early diagnosis of vestibular schwannoma is essential to disease prognosis. Physicians must be able to recognize suggestive clinical signs, and the index of suspicion should be particularly high in patients just past the age of 40 . Vestibular schwannoma must be ruled out in all patients presenting with sensorineural hearing loss, particularly when the hearing loss is asymmetric.

Complete removal of vestibular schwannomas is only possible by means of surgery, the only treatment modality that permits resection of the entire tumor with little chance of recurrence.

Other therapeutic options include watchful waiting ("wait and scan"), in very small tumors (Class I), very old patients, or those who are poor candidates for surgery and exhibit no major brainstem compression.

Stereotactic surgery $(20,21)$ is indicated in class I or II tumors and in select other cases. This treatment modality provides an attempt at halting tumor growth with a substantial disadvantage of severely jeopardizing the surgical field if future intervention is required. Although rare, facial palsy and hearing loss have been reported as complications.

The outcomes of vestibular schwannoma surgery have improved considerably with advances in diagnosis.
In the early 20th century, when only large tumors were diagnosed, the main measure of surgical success was achieving complete or partial tumor resection without causing the death of the patient. At the time, surgical mortality was extraordinarily high. The first successful resection of vestibular schwannoma was performed in 1894 by Charles Ballance, through the suboccipital route (22).

As improvements in technique enabled early diagnosis, the main concern of surgeons shifted to preservation of facial nerve function.

In 1904, Panse described the translabyrinthine approach to the cerebellopontine angle (23), which was, however, (initially) soon abandoned due to a lack of adequate surgical instruments and to the difficulty of tumor visualization in the pre-microscopic age.

After 1920, the translabyrinthine approach appeared destined to oblivion. Only in 1961, after operating with microsurgical instruments and the operating microscope had become routine in otologic surgery, and more effective methods had been made available for diagnosis of these tumors, did House restore the translabyrinthine route to prominence, achieving a mortality rate of $5.4 \%$ and $95 \%$ preservation of the facial nerve, and completely changing the surgical prognosis of this condition by a dramatic reduction in morbidity and mortality and substantial improvement in quality of facial nerve function $(24,25,26)$.

Preservation of facial nerve integrity is now possible in the large majority of cases, and is directly associated with tumor size. More refined surgical techniques, with the advent of more appropriate instruments, intraoperative monitoring of facial and auditory nerve function, and ultrasonic aspirators, have made operative approaches to these tumors much safer. The most significant challenge now is preservation of hearing without detriment to the main goals of surgery, which are low morbidity and mortality, complete tumor resection, and preservation of the facial nerve.

Vestibular schwannoma surgery has been the object of numerous publications by otorhinolaryngologists and neurosurgeons, with vigorous debate on routes of access, intraoperative and postoperative complications, residual tumor tissue and tumor recurrence after resection, and preservation of hearing. With some variation, there are 5 broad approaches that are used for resection of vestibular schwannomas, each with its particular pros and cons. These approaches to the internal acoustic canal and cerebellopontine angle can be divided into presigmoid, retrosigmoid, middle fossa, supratemporal, and combined. 
The presigmoid routes are the translabyrinthine approach and the retrolabyrinthine approach.

The retrosigmoid routes are the suboccipital approach and the posterior fossa approach.

The supratemporal routes are the middle fossa approach and the extended middle fossa approach, and combined approaches are a combination of any of the aforementioned routes.

The objective of this article is to report the signs and symptoms exhibited in 825 cases of vestibular schwannoma treated surgically between 1981 and 2006, describing the relevant aspects of clinical, audiometric, and imaging diagnosis; the operative techniques used in this series; and perioperative and postoperative complications, thereby proposing a standardized methodology for diagnosis and treatment.

\section{Case Series and Methods}

A detailed retrospective chart review was conducted of 825 patients diagnosed with vestibular schwannoma who underwent surgery between January 1984 and August 2006. Resection was performed systematically by the same surgeon and team. Most patients had been referred for surgery after a definitive diagnosis had been established by other clinicians at our service or elsewhere.

All patients underwent a new history and physical and complete ear, nose, throat, and neurological examination, as well as the following examinations:

- Pure tone audiometry

- Speech audiometry with single-syllable and spondaic words for determination of the Speech Recognition Index

- Auditory brainstem response (ABR) audiometry, when thresholds so permitted

- Computed tomography (CT) of the temporal bone

- Magnetic resonance imaging (MRI) of the head.

The following data, obtained by means of clinical history, were tabulated for the purposes of this study:

- Age at time of diagnosis

- Sex

- Affected side

- Chief complaint

- Duration of chief complaint

- Tumor size

- Other signs and symptoms.

Inquiries made of patients were regarding the following associated signs and symptoms: hearing loss; continuous or intermittent tinnitus; vertigo; dizziness or unsteadiness; headache; facial pain or hypoesthesia, including hypoesthesia of Ramsey Hunt's zone or the external auditory meatus; earache; presence of Hitselberger's sign; facial palsy; facial spasm; aural fullness; eye pain; signs of intracranial hypertension; and neuralgia.

Audiometric findings were classified as follows:

- Pure tone audiometry (for the frequencies 250, 500, 1000, 2000, 4000, and $6000 \mathrm{~Hz}$ )

- Profound hearing loss: $>90 \mathrm{~dB} \mathrm{HL}$

- Severe hearing loss: $>70 \mathrm{~dB}$ HL

- Moderate hearing loss: 51-70 dB HL

- Mild hearing loss: 26-50 dB HL

- Normal hearing: down to $25 \mathrm{~dB}$ HL

Tumor size was classified by the grading scheme described in the introduction section, based on MRI findings that were clinically correlated with hearing thresholds, tumor size classification, and speech recognition index.

The following surgical approaches were employed throughout our 25-year experience:

- Translabyrinthine.

- Retrolabyrinthine.

- Middle fossa.

- Combined posterior fossa and translabyrinthine (24).

\section{RESULTS}

Of the patients included in the sample, 467 (56.6\%) were female and 358 (43.4\%) were male. Regarding laterality, 398 (48.2\%) tumors were right-sided and 427 (51.8\%) were left-sided.

The chief complaint was progressive unilateral hearing loss in $656(79.5 \%)$ patients, tinnitus in 67 (8.1\%), and sudden hearing loss in 48 (5.8\%). Vertigo (5.1\%), facial palsy (1\%), hemifacial spasm (0.4\%), and decreased sensitivity of the internal auditory canal (0.1\%) were less frequent complaints (Chart 1).

Regarding the duration of the chief complaint at diagnosis, 94 (11.4\%) patients had had the symptom for less than 6 months, 170 (20.6\%) for 6 months to 1 year, and $561(68 \%)$ for more than 1 year (Chart 2).

Patient ages at diagnosis were distributed as follows (Chart 3):

0-20 years: $12(1.5 \%)$

21-30 years: $55(6.7 \%)$

31-40 years: $108(13.1 \%)$

41-50 years: $329(39.8 \%)$

51-60 years: $216(26.2 \%)$

61-70 years: $82(9.9 \%)$

71-80 years: $23(2.8 \%)$. 
Other signs and symptoms found in association with the chief complaint are shown in Chart 4.

With respect to hearing loss on the affected side, at the time of surgery, 220 patients (26.7\%) had profound hearing loss, 261 (31.6\%) had severe hearing loss, 279 (33.8\%) had moderate hearing loss, 53 (6.4\%) had mild hearing loss, and 12 (1.5\%) had normal thresholds.

The spondee recognition score on the affected side was 100\% in 146 patients (17.7\%), 70-99\% in 212 patients (25.7\%), 50-69\% in 241 patients (29.2\%), 30-49\% in 97 patients (11.8\%), and $<30 \%$ in 129 patients.

Electronystagmography was performed in $511 \mathrm{ca}-$ ses. Results were as follows:

- Normal: 161 (31.5\%)

- Ipsilateral hyporeflexia: 97 (19\%)

- Contralateral hyporeflexia: 67 (13.1\%)

- Ipsilateral hyperreflexia: 45 (8.8\%)

- Contralateral hyperreflexia: 65 (12.7\%)

- Areflexia: 34 (6.5\%)

- Signs of central involvement: 42 (8.2\%).

ABR audiometry was not performed due to low hearing thresholds in 333 patients (40.4\%). In the remaining patients, it revealed signs of retrocochlear dysfunction in $352(42.7 \%)$ and was within normal limits in 29 (3.5\%). There were no data on ABR in the charts of 111 patients (13.4\%).

Tumor size on MRI was consistent with Grade I in 189 cases (22.9\%), Grade II in 401 (48.6\%), Grade III in 188 (22.8\%), and Grade IV in 47 (5.7\%), according to the classification scheme described in the introduction section.

Percentage correlations between tumor size and auditory involvement are shown in Chart 5.

Regarding the surgical route of choice, the translabyrinthine approach was employed in 704 patients (85.3\%), the retrolabyrinthine in 93 (11.3\%), the middle fossa approach in $6(0.7 \%)$, and the combined approach in 22 cases $(2.7 \%)$.

Complete resection was achieved in 813 cases (98.5\%), and planned partial resection in 12 (1.5\%).

Early postoperative complications (occurring within 1 month of surgery) included CNS fistula in 46 patients (5.5\%), intracranial hypertension in 8 (1\%), intracranial hemorrhage in $6(0.9 \%)$, cerebellar syndrome in $6(0.9 \%)$, and meningitis in $45(5.4 \%)$. There were 4 deaths $(0.5 \%)$.

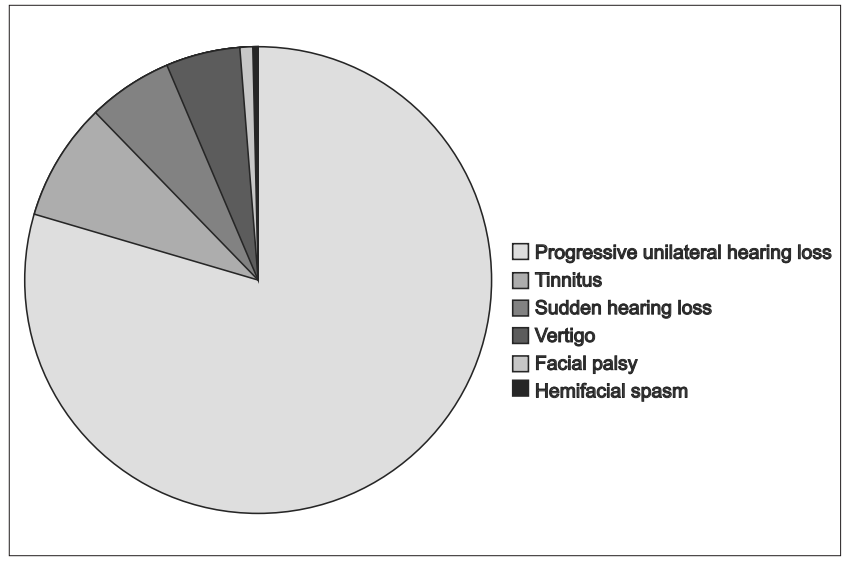

Chart 1.

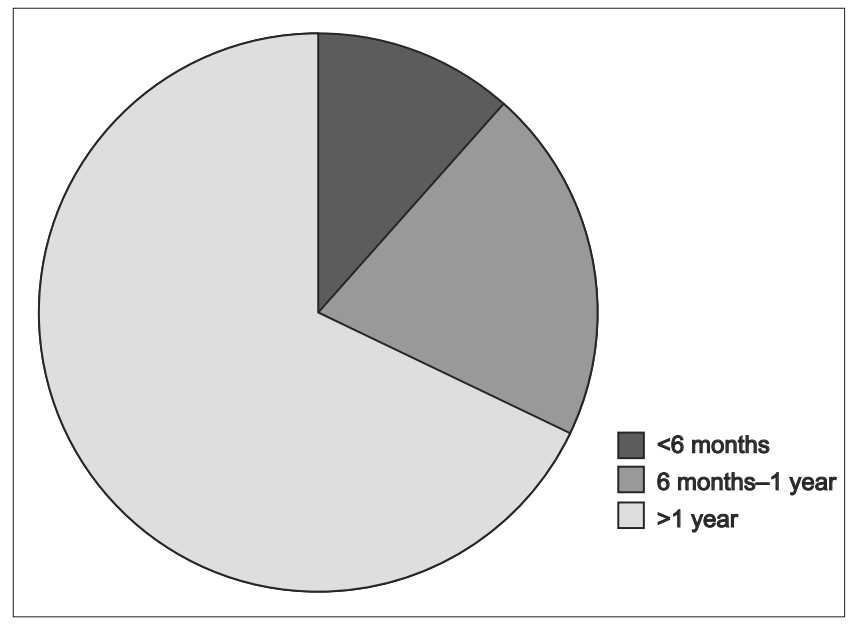

Chart 2.

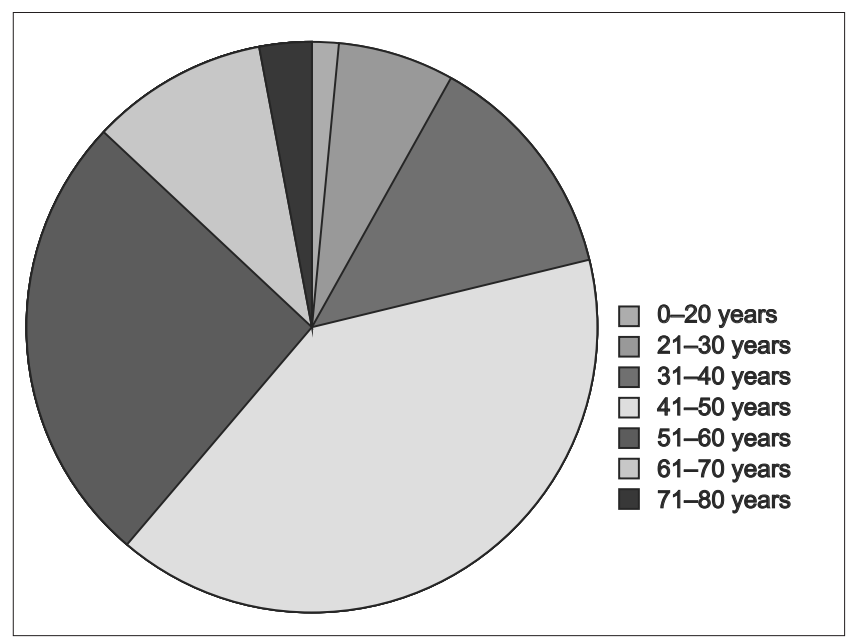

Chart 3. 


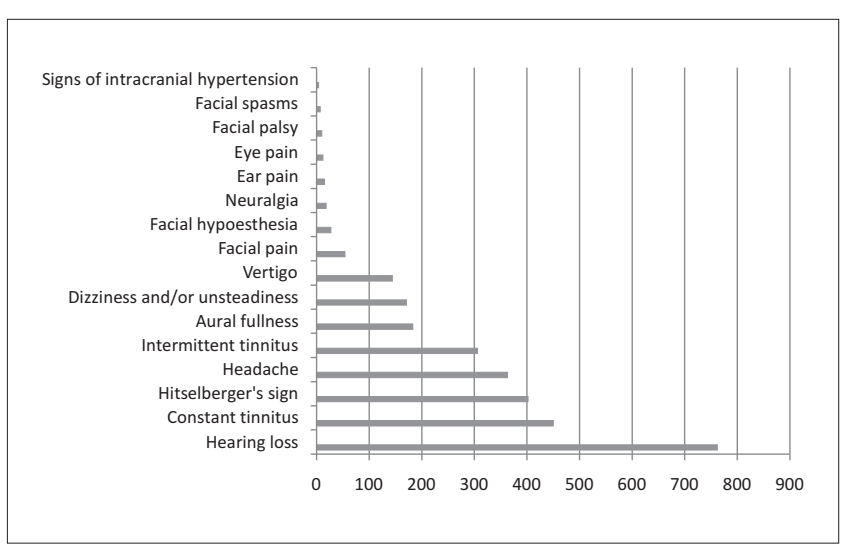

Chart 4. Signs and symptoms.

Facial nerve function on the tenth postoperative day (House-Brockmann score) (25) was grade V or VI in 167 patients (20.2\%), grade II, III, or IV in 212 (25.7\%), and Grade I in 446 (54.1\%). At 16-month follow-up, function was normal in 617 patients (74.8\%), Grade II in 89 (10.8\%), Grade III in 27 (3.3\%), Grade IV in 25 (3\%), Grade V in 36 (4.4\%), and Grade VI in 31 (3.7\%).

In the 93 patients in whom the retrolabyrinthine approach was employed, auditory function at 90-day follow-up (as represented by audiometric thresholds) was unchanged in 45 (48.4\%), worse in 7 (7.5\%), and had progressed to complete deafness in 41 (44.1\%). In patients with preserved hearing, speech discrimination was unchanged in 40 cases (88.9\%) and worse in 5 (11.1\%).

After a minimum 5-year follow-up, there were no signs of recurrence or residual tumor in 813 cases (98.5\%). Residual tumor remained in the 12 cases in which partial resection had been planned (1.5\%)

\section{DISCUSSION}

\section{Auditory Signs and Symptoms}

According to most authors, progressive, unilateral hearing loss is the initial symptom in approximately $90 \%$ of vestibular schwannomas. In our sample, it was the chief complaint in roughly $80 \%$ of cases. Hearing loss may present or worsen suddenly, as observed in $6 \%$ of cases in our series. According to Sauvaget, 3 to 23\% of vestibular schwannomas produce sudden hearing loss, whereas only $2 \%$ of cases of sudden hearing loss lead to a diagnosis of schwannoma (19).

The degree of hearing loss is not always associated with the anatomical stage of the disease, as shown by our

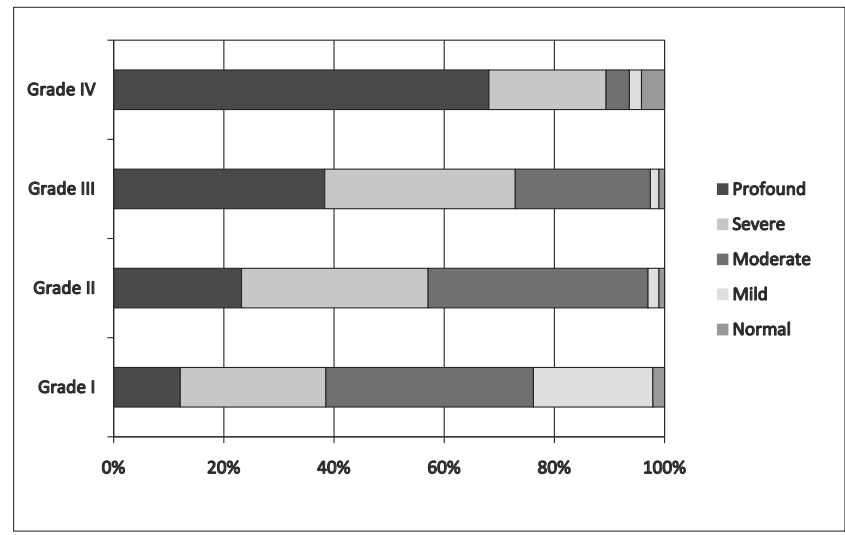

Chart 5. Correlations between tumor size and auditory involvement.

assessment of the correlation between tumor size and audiometric thresholds and speech discrimination. Analysis of these findings shows that, although larger tumors are generally associated with greater involvement of auditory thresholds, there are cases of small tumors with major auditory involvement and, conversely, large tumors with little effect on auditory function were present in all 3 groups.

Analysis of speech discrimination more clearly demonstrates that involvement of this parameter is not proportional to tumor size.

In this series, most patients were diagnosed between the ages of 41 and 60 (66\%) years, which is consistent with the international literature.

Sixty percent of patients were women, which is also consistent with reports of a female preponderance.

Aural fullness is an infrequent symptom. It was not the chief complaint in any of the patients in this series, but was reported in $22.2 \%$ of clinical histories. This symptom is often associated with fluctuating hearing loss.

In older patients, vestibular schwannoma can be mistaken for labyrinthine hydrops or vascular dysfunction, and this can be an important cause of diagnostic delays.

Tinnitus, usually high-pitched, was the second most common isolated symptom in our series (8.1\%). Continuous or intermittent tinnitus with concomitant hearing loss is present in approximately 90\% of cases, and is nearly always unilateral.

Vertigo, usually positional, is the presenting symptom in only $5 \%$ of patients. As an associated, but not isolated complaint, balance disorder ranging from unsteadiness to frank rotational vertigo is reported by $40 \%$ of patients. In 
the literature, reports of balance disorder in vestibular schwannoma vary widely. Some authors maintain it may be the presenting symptom in 15\% of cases.

Headache (40\%), facial pain, and earache are also reported, strictly as isolated symptoms. Facial palsies, spasms and dysesthesias, eye pain, and signs of intracranial hypertension are even less frequent, and may reflect advanced-stage or complicated disease.

Subjective reports of trigeminal nerve dysfunction are limited to a feeling of thickening of the cheek area, but the entire trigeminal zone may be affected. Trigeminal neuralgia may occur, usually in combination with somewhat diminished facial sensitivity, but it is rare and not characteristic of vestibular schwannoma.

\section{Facial nerve involvement}

A review of the literature shows that, on average preoperative functional involvement of the facial nerve is present in $10 \%$ to $30 \%$ of vestibular schwannomas. In our series, only $2.2 \%$ of patients exhibited such involvement, and the palsy was incomplete, a paresis presenting as barely visible asymmetry of the lower face muscles, flattening of the nasolabial fold evident only during facial movements, and no apparent loss of strength or symmetry during forced movement. Functional exploration of the facial nerve (stimulation, latency measurement, electromyography) might reveal subclinical involvement, but these data were missing for most patients in the present series and were therefore not taken into account.

\section{Nervus intermedius involvement}

Changes involving the nervus intermedius (nerve of Wrisberg) may present as an isolated symptom or in combination with taste disturbance(due to increased electrogustometry thresholds in the anterior two-thirds of the tongue) (not observed in our series), reduced tear secretion on Schirmer's test (not observed in our series), defective nasolacrimal reflex (not elicited in most of our patients), and reduced sensitivity in Ramsey Hunt's zone. The latter sign was the presenting symptom of vestibular schwannoma in 1 case in our series, occurring in combination with the chief complaint in $9.5 \%$ of cases, and was found on physical examination in $48.7 \%$ of patients. This is an extremely important finding, as Hitselberger's sign was positive in nearly $50 \%$ of patients in this series, making it a very useful component of the physical examination, in fact, much more useful than many tests often heralded as valuable in diagnosis of vestibular schwannoma, including electronystagmography, which showed absolutely no evidence of diagnostic utility in our series.

Clinical symptoms of CN IX, X, XI, or XII dysfunction are exceedingly rare in this disease. Dysphonia and dysphagia (suggesting IX and X involvement) are observed in some cases, and are always indicative of advanced tumor development and inferior extension. None of our patients exhibited these symptoms.

Headache is commonly reported ( $44 \%$ of cases in this series), and is usually caused by large tumors. Later in the disease course, headache may signal intracranial hypertension. As an isolated symptom, it is difficult to assess, as headache has so many other causes and is one of the most commonly reported of all symptoms.

\section{Cerebellarinvolvement}

Cerebellar incoordination is rare and presents essentially as ataxia ipsilateral to the tumor. No cases were found in this series.

All symptoms and signs are represented to varying degrees in the medical literature, due to major country-tocountry variability in study samples, the possibility of more advanced diagnostic modalities in some centers, earlier and more universal access to health care depending on location, varying awareness of medical conditions among the population, and differences in the training of general practitioners and specialists with regard to whether cerebellar schwannoma should always be ruled out in patients with a history of hearing loss.

\section{General physical examination findings}

In patients with neurofibromatosis type 2, the physical examination may reveal a variety of findings, including neurofibromata throughout the body and café au lait spots. These patients may have bilateral vestibular schwannomas. In our series, 3 patients had bilateral tumors. All were classified as having neurofibromatosis type 2 .

With respect to audiological testing, pure tone audiometry remains the essential modality. Interest in classical above-threshold ("supraliminal") tests and Békésy audiometry has waned since the advent of impedance audiometry and evoked potential testing.

In a broad review by several authors (Brackmann, Sterkers, Portmann), the mean pure-tone threshold at 500, 1000, 2000, and $4000 \mathrm{~Hz}$ was $72 \mathrm{~dB}$, not taking into account disease progression. 
Mean thresholds for each disease stage were as follows:

- $61 \mathrm{~dB}$ for intracanalicular tumors

- $73 \mathrm{~dB}$ for stage II tumors

- $71 \mathrm{~dB}$ for stage III tumors

- $74 \mathrm{~dB}$ for stage IV tumors.

The most commonly encountered audiometric picture is an abrupt decline (audiometric notch or dip) at $4000 \mathrm{~Hz}$, with or without $>25 \mathrm{~dB}$ hearing loss at frequencies normally used in conversation.

\section{Speech audiometry}

Assessment of speech discrimination is essential in the diagnostic workup of vestibular schwannoma, and is abnormal in most cases. In our series, $72.6 \%$ had a speech recognition index of $50 \%$ or higher. The relationship between speech discrimination and tumor size is unclear.

Patients with acoustic neurinoma develop a vestibular deficiency syndrome. This deficit occurs gradually and is masked by the adaptive abilities of the vestibular system. Vestibular assessment may include positional maneuvers and caloric testing.

In our series, otoneurological examination was performed in patients who reported balance disorders, but results were inconsistent and did not elicit any clear diagnostic suspicion. Furthermore, the otoneurological examination has been superseded by brainstem auditory evoked potential (BAEP) testing for diagnosis of vestibular schwannoma. In our opinion, electronystagmography as an isolated test should not be used routinely as part of the diagnostic workup when vestibular schwannoma is suspected.

\section{Diagnostic Imaging}

Imaging tests have become essential for definitive diagnosis, and should always be performed when there is clinical or audiological suspicion of cerebellopontine angle disease. Imaging allows diagnosis of cerebellopontine angle masses and estimations of size and provides information on potential effects on CSF dynamics.

MRI is undoubtedly the imaging modality of choice when cerebellopontine angle disease is suspected. Gadolinium contrast-enhanced MRI has nearly 100\% reliability, even for small tumors. Schwannomas exhibit intermediate signal intensity on $\mathrm{T} 1$ and marked enhancement during the contrast phase. On T2-weighted images, the tumor may not be visible or may present as signal suppression. T2 sequences are also useful for assessment of the relationship between the tumor and the internal auditory canal, inner ear, and adjacent cranial nerve pairs.

On the basis of these results, our current protocol for diagnosis of cerebellopontine angle masses is as follows:

- Clinical history and audiometric evidence of sensorineural hearing loss, particularly when asymmetric:

- BAEP and contrast-enhanced MRI. If BAEP is prohibitively expensive or might lead to a delay in diagnosis, MRI alone is performed. Once the diagnosis has been established, contrast-enhanced CT of the temporal bone, with particular emphasis on the internal auditory canal, is required for surgical planning. Determination of the spatial relationship between the jugular bulb and the internal auditory canal is especially important.

- Physicians should never neglect signs or symptoms indicative of vestibular schwannoma, particularly sensorineural hearing loss (regardless of frequency), unilateral tinnitus, or vestibular hyporeflexia. In patients with these findings, all diagnostic possibilities should be exhausted, as early tumor detection ensures the best possible prognosis with surgical treatment.

\section{Treatment}

In the 825 consecutive cases included in this series, the surgical techniques employed were effective in enabling complete tumor resection, except in 12 patients with bilateral schwannomas due to neurofibromatosis. In these patients, partial resection was planned with the purpose of decompressing the brainstem, which was affected by tumor encroachment, and preserving hearing and facial nerve function as long as possible, as bilateral disease invariably leads to dysfunction as it progresses.

Early postoperative complications occurred in $13.3 \%$ of cases, and included CNF fistula (5.5\%), which was treated with continuous lumbar puncture over 3 days, compression dressings, and treatment of meningitis, which occurred in all cases. In all patients, closure of the fistula was achieved, infection was managed successfully, and surgical outcomes remained unchanged.

There were patients with clinical and CT evidence of intracranial hypertension (1\%) or intracranial bleeding $(0.9 \%)$ who underwent reoperation via the retrosigmoid route to good effect. These patients had Grade III tumors; in 2 patients, a combined retrosigmoid and middle fossa approach was employed. The mortality rate in this series was $0.5 \%$ ( 4 deaths). These findings indicate the striking improvements in the prognosis of surgery for vestibular schwannoma in Brazil since as recently as the 1970s. 
In 90\% of cases, facial nerve function, which we believe to be the most important factor after complete resection and absence of general complications, was preserved to House-Brackmann Grades I, II, and III, with Grade I (normal) function in $74 \%$ of cases, providing evidence of the high effectiveness of current techniques for preservation of the facial nerve. Total palsy occurred in $3.7 \%$ of cases, and more severe dysfunction developed in patients with large (Grade III or IV) tumors, who required placement of nerve grafts in the posterior fossa. Facial nerve involvement is most extensive in the early postoperative period and improves over 1 year, which indicates that initial dysfunction merely reflects reversible paresis produced by surgical manipulation of the nerve.

One of the most encouraging findings of our study is that auditory function was preserved in 50\% of patients who underwent surgery via the retrolabyrinthine approach. We believe this route represents the future of vestibular schwannoma surgery with regard to preservation of hearing.

\section{CONCLUSIONS}

1) In nearly all cases, asymmetric sensorineural hearing loss is the first symptom of vestibular schwannoma.

2) Tumor size is not proportional to the extent of hearing threshold and speech discrimination involvement.

3) In this sample, magnetic resonance imaging was the modality of choice for definitive diagnosis.

4) The translabyrinthine approach was safe and resulted in few intraoperative or postoperative complications, all of which were managed, and can be used alone or as part of a combined approach to very large tumors.

5) All techniques employed in this series were safe with regard to preservation of facial nerve function.

6) The retrolabyrinthine approach was useful in small tumors, with 50\% preserved hearing, and can be recommended in patients with good thresholds and word discrimination.

\section{REFERENCES}

1. Woellner RC, Schuknecht HF. Hearing loss from lesions of the cochlear nerve: an experimental and clinical study. Trans Am Acad Ophthalmol Otolaryngol. 1955;59 (2):1479 .

2. Stewart TJ, Liland J, Schuknecht HF. Ocult schwannomas of the vestibular nerve . Arch. Otoloryngol. 1975;110(2):9195.

3. Sandifort E. Observationes anatomicae-pathologicae, vd Eryck P, Vygh D, Lugduni Batavorum 1777.
4. Bento RF, Miniti A, Marone SAM. Tratado de Otologia. São Paulo, EDUSP, 1998.

5. Cushing H. Tumors of the nervus acusticus and the syndrome of cerebellopontine angle, Philadelphia, Saunders, edit., 1917.

6. Revilla AG (1948), apud Dykstra PC (1964).

7. Dykstra PC. The pathology of acoustic neuromas. In: House WF. Monograph- Transtemporal Bone Microsurgical Removal of Acoustic Neuromas. Arch Otolaryngol. 1964;80:751-2.

8. House WF. Transtemporal bone microsurgical removal of acoustic neuromas: report of cases. Arch. Otolaryngol. 1964;80:617-667.

9. Pulec JL, House W, Britton Jr BH, Hitselberger WE. - A system of management of acoustic neuroma based in 364 cases. Trans Am Acad Ophtalmol. Otolaryngol.; 1971;75:4855.

10. Schoemaker MJ, Swerdlow AJ, Ahlbom A, Auvinen A, Blaasaas K, Cardis E, Christensen HC, Feychting M, Hepworth SJ, Johansen C, Klaeboe L, Lonn S, Mc Kinney PA, Muir K, Raitanen J, Salminen T, Thomsen J, Tynes T. Mobile phone use and risk of acoustic neuroma: results of the interphone case-control study in five North European countries. BR J Cancer. 2005;93(7):842-8.

11. Takebayashi T, Akiba S, Kikuchi Y, Taki M, Wake K, Watanabe S, Yamaguchi N. Mobile phone use and acoustic neuroma risk in Japan. Occup Environ Med. 2006;63 (12):802-7.

12. Tato JM, Venturini N, Ganança M, Anicet A, AntoniCandela F, Linden A. Diagnóstico e tratamento do neurinoma do acústico. Rev Bras Otorrinolaringol. 1970; 36:107-117.

13. Souza OG, Inácio AA, Cabral FG, Carneiro FA, Nunes CA. Neurinoma do Acústico. Rev Bras Otorrinolaringol. 1974;40:157-161.

14. Souza OG, Santos SP, Inácio AA, Barbosa VC, Cabral FG. Otoneurocirurgia do meato acústico interno. Rev Bras Otorrinolaringol. 1974;38:200-206.

15. Souza NJA, Guimarães RES, Guimarães HA, Cadar A, Coelho CEC. Neurinoma do Acústico. Rev Bras Otorrinolaringol. 1976;42:35-37.

16. Bento RF, Caropreso CA, Miniti A. A via translabiríntica na cirurgia do neurinoma do acústico. Rev Bras Otorrinolaringol. 1989;55:57-63. 
17. Bento RF, Brito RV, Sanchez TG, Miniti A. The transmastoid retrolabyrinthine approach in vestibular schwannoma surgery. Otolaryngol Head Neck Surg. 2002;127 (5):437-41.

18. Souza OG, Siqueira JM. Conclusões e recomendações da 1a. Conferência Internacional de neuroma do acústico. Rev Bras Otorrinolaringol. 1992;58:257-259.

19. Sauvaget E, Kici S, Kania R, Herman P, Tran Ba Huy, P. Sudden Sensorioneural hearing loss as a revealing symptom of Vestibular Schwanoma. Acta Otolaryngol. 2005;125(6):592-5.

20. Linskey ME, Lunsford LD, Flickinger JC. Radiosurgery for acoustic neuromas: early experience. Neurosurgery 26:736-745, 1990

21. Flickinger JC, Lunsford LD, Coffey RJ. Radiosurgery for acoustic neurinomas. Cancer. 1991;67:345-353.
22. Ballance C (1907), apud House WF. Transtemporal bone microsurgical removal of acoustic neuromas: report of cases. Arch. Otolaryngol. 1964;80:617-667.

23. Panse D (1904), apud House WF. Transtemporal bone microsurgical removal of acoustic neuromas: report of cases. Arch. Otolaryngol. 1964;80:617-667.

24. Hitselberger WE, Pulec JL. Trigeminal nerve (posterior root) retrolabirintine, transtentorial, approach to the brainstein. Otolaryngol Head Neck Surg. 1991;104:130-131.

25. House JW, Brackmann DE. Facial nerve grading system. Otolaryngol. Head Neck Surg. 1985;93:146-147.

26. Yasargil MG. Legacy of microneurosurgery: memoirs, lessons, and axioms. Neurosurgery. 1999;45:1025-91. 